\title{
¿Es saludable una dieta vegana? Un dilema para desarrollar el pensamiento crítico a través de la argumentación y la toma de decisiones en la formación inicial de maestros
}

\author{
José Manuel Hierrezuelo-Osorio, Vito-Battista Brero-Peinado \\ y Antonio Joaquín Franco-Mariscal \\ Universidad de Málaga. Didáctica de las Ciencias Experimentales.
}

[Recibido el 10 de mayo de 2020, aceptado el 1 de julio de 2020]

\begin{abstract}
El desarrollo del pensamiento crítico en la formación inicial del profesorado es un tema relevante en la enseñanza de las ciencias. Este trabajo explora cómo abordarlo en la enseñanza universitaria utilizando el dilema como estrategia didáctica para potenciar la argumentación y la toma de decisiones. Se presentan los resultados de un dilema sobre si la dieta vegana es saludable o no implementado con 191 estudiantes de los Grados en Educación Infantil y Educación Primaria de la Universidad de Málaga. La influencia de la actividad se analiza a través de las habilidades de argumentación incluidas en un cuestionario más amplio de pensamiento crítico respondido a modo de pre-test y post-test. La comparación de los valores medios de dichas habilidades revela una ligera mejora del pensamiento crítico en ambos grados. De forma mayoritaria, los estudiantes consideran que la dieta vegana no es saludable, y son poco reacios a cambiar su decisión inicial. Asimismo, se comprueba que la última información recibida influye en la decisión adoptada.
\end{abstract}

Palabras clave: pensamiento crítico; maestros formación inicial; educación para la salud; dieta vegana.

\section{Is healthy a Vegan Diet? A Dilemma to Develop Critical Thinking through Argumentation and Decision-Making in Initial Teacher Training}

The development of critical thinking in initial teacher training is a relevant topic in science education. This paper explores how to address it in university education by using the dilemma as an educational strategy to enhance argumentative and decision-making skills. The results of a dilemma about the vegan diet implemented with 191 students of the Early Childhood Education and Primary Education Degrees of the University of Málaga are presented. The influence of the activity is analysed through the argumentation skills included in a broader critical thinking questionnaire answered as a pre-test and a post-test. The comparison of the mean values of these skills reveals a slight improvement in critical thinking in both grades. Most students consider the vegan diet unhealthy being unwilling to change their initial decision. Also, the latest information received influences on the decision.

Keywords: critical thinking; pre-service teachers; health education; vegan diet

Para citar el artículo. Hierrezuelo-Osorio, J.M., Brero-Peinado, V.B. y Franco-Mariscal, A.J. (2020). ¿Es saludable una dieta vegana? Un dilema para desarrollar el pensamiento crítico a través de la argumentación y la toma de decisiones en la formación inicial de maestros. Ápice. Revista de Educación Científica, 4 (2), 73-88. DOI: https://doi.org/10.17979/arec.2020.4.2.6525

Contacto. jose.hierrezuelo@uma.es 


\section{Introducción}

En los últimos años, la educación está teniendo un papel clave por la repercusión que tiene en el futuro de sus estudiantes, ya que el objetivo es que se conviertan en ciudadanos competentes y responsables con un pensamiento crítico que haga frente a los retos actuales de la sociedad. El pensamiento crítico se define como el pensamiento racional y reflexivo enfocado a decidir qué creer o hacer, y abarca ciertas destrezas o habilidades y disposiciones (Ennis, 1991; Cobo, Abril y Romero, 2018).

El desarrollo del pensamiento crítico de los futuros docentes se considera una competencia profesional importante ya que incentiva una educación donde sean protagonistas, se formen como ciudadanos libres y les permita construir conocimientos científicos coherentemente (Solbes y Torres, 2012). Asimismo, el grado de adquisición de esta competencia en la formación inicial de docentes está relacionado con su capacidad para fomentarla con mayor o menor éxito en sus estudiantes.

El desarrollo de habilidades de pensamiento crítico debe ser entendido como una herramienta útil para comprender y explicar lo que está ocurriendo a nuestro alrededor (Fonseca y Castiblanco, 2020). De hecho, las mejoras producidas en el pensamiento crítico de los estudiantes influirán directamente en tres aspectos fundamentales, como son, la mejora en las habilidades implicadas, la asimilación y adopción de estas mejoras durante tiempo prolongado, y la posible extrapolación a otros contextos (Manassero y Vázquez, 2020).

Sin embargo, la formulación de los actuales currículos en torno al desarrollo del pensamiento crítico plantea desafíos sobre cómo llevarlas al aula. De hecho, son los propios docentes en formación inicial (Solbes y Torres, 2013) los que ponen de manifiesto la necesidad de una articulación de las asignaturas en relación con el pensamiento crítico ya que resaltan una casi exclusiva transmisión de contenidos, como conceptos y fórmulas, y que difícilmente se desarrollan estrategias u actividades que promuevan el pensamiento crítico debido, principalmente, a razones relacionadas con la falta de un contexto educativo propicio, a los propios estudiantes, a su propia falta de formación en esta habilidad y a una enseñanza unidimensional de la ciencia.

Por una parte, el docente debe plantearse qué contenidos enseñar, y tener conciencia de su pertinencia, además de saber qué tipo de lenguaje usar en su clase para comunicar esta ciencia a sus estudiantes, mientras que por otra parte, a los estudiantes se les debe potenciar la autonomía, lo que hace necesario una formación en participación activa, donde puedan expresarse, opinar, cooperar en el aula y desarrollar su propia perspectiva sobre un determinado tema (Fonseca y Castiblanco, 2020).

Además, debemos tener en cuenta que los maestros en formación inicial están aprendiendo el oficio de docente, por lo que tienen que conocer y practicar de primera mano enfoques y estrategias que se espera que utilicen en su práctica profesional. En este sentido, las propuestas que se realicen en el aula deben formularse desde una concepción del docente como práctico reflexivo (Blanco, España y Franco-Mariscal, 2016), capaz de construir conocimiento a partir de su implicación personal y profesional.

En un trabajo reciente, Blanco, España y Franco-Mariscal (2017) propusieron diferentes estrategias didácticas útiles para desarrollar el pensamiento crítico en ciencias, entre las que destacan la argumentación científica y la toma de decisiones ante problemas socio-científicos. El desarrollo del pensamiento crítico a través de la argumentación supone crear argumentaciones sólidas y cuestionar la validez de los argumentos, rechazando conclusiones no basadas en pruebas, y detectando falacias argumentativas. La toma de decisiones supone hacer elecciones racionales y juicios fundamentados como elementos de las decisiones que emplean para resolver problemas. 
Para estos propósitos, el uso de dilemas o cuestiones socio-científicas se presenta como una estrategia valiosa para tratar en el aula cuestiones relacionadas con el medioambiente, la salud, la energía, etc. y desarrollar habilidades de pensamiento crítico (Evagorou, JiménezAleixandre y Osborne, 2012; Simonneaux y Lipp, 2017). Su tratamiento implica el uso de la argumentación para la toma de decisiones, entendiéndose esta habilidad como la capacidad para evaluar enunciados en base a pruebas, y reconociendo que las conclusiones y los enunciados científicos deben ser justificados y sustentados en estas pruebas (JiménezAleixandre, 2010). El empleo de estas cuestiones socio-científicas favorecen también el aprendizaje de conocimientos científicos necesarios para que los ciudadanos puedan comprender diferentes riesgos y dilemas que surgen en su vida diaria (Díaz y Jiménez-Liso, 2012), a la vez que ayudan a mejorar la comprensión conceptual, la indagación científica, las actitudes y los valores sociales (Evagorou, Jiménez-Aleixandre y Osborne, 2012).

El objetivo de este trabajo es presentar los resultados de una actividad llevada a cabo con maestros en formación inicial, incluida dentro un programa formativo más amplio sobre pensamiento crítico, centrada en un dilema actual en la sociedad relacionado con hábitos de vida saludable, como la dieta vegana, y que permite abordar la argumentación y la toma de decisiones fundamentada como habilidades esenciales en el desarrollo del pensamiento crítico. Estos resultados nos permiten valorar la estrategia de instrucción y la evolución global que este tipo de actividades produce en el desarrollo del pensamiento crítico.

\section{Metodología}

\section{Contexto}

El presente estudio se enmarca en un programa formativo para desarrollar habilidades de pensamiento crítico desde la formación inicial de profesorado. Dicho programa se está llevando a cabo con 191 estudiantes de la Universidad de Málaga, de edades comprendidas entre 20 y 43 años, matriculados en asignaturas de Didáctica de las Ciencias Experimentales del tercer curso de los Grados en Educación Infantil (GI) (N=111) y en Educación Primaria (GP) ( $\mathrm{N}=80$ ). El programa formativo fue desarrollado en cada grado por un docente diferente. Ambos docentes (primer y tercer autores del artículo) son investigadores en Didáctica de las Ciencias Experimentales y diseñaron de forma conjunta el programa. Asimismo realizaron un seguimiento de la implementación de forma coordinada, para que la puesta en práctica se desarrollara en las mismas condiciones.

\section{Breve descripción del programa formativo}

Con la intención de que el lector se haga una idea del contexto en el que se encuadra la actividad que posteriormente se presenta, este apartado describe de forma breve el programa formativo en el que se realizó. Dicho programa consta de dos partes. En la primera parte, los estudiantes adquirieron un papel de receptores de información e instrucción, mientras que en la segunda asumieron el rol de maestros, con objeto de explorar la posible transferencia al aula. A su vez, dentro de estas dos partes, se establecen cuatro fases (figura 1), que se describen seguidamente.

Fase 1: Administración de pre-test para identificar el estado inicial de pensamiento crítico de los estudiantes a través de la argumentación.

Este pre-test consta de un cuestionario para identificar el estado inicial de pensamiento crítico de los estudiantes propuesto y validado por Santiuste et al. (2001) y de dos actividades. En la primera actividad se suministran a los estudiantes titulares de prensa correspondientes a varios dilemas de carácter socio-científico, a partir de los cuales se les pide 
elaborar argumentos a favor y en contra de los mismos y tomar una decisión al respecto. A continuación, en la segunda actividad se les vuelven a administrar los mismos titulares de prensa, pero, en este caso, acompañados con información detallada para cada titular en distinto formato (texto, vídeo, opiniones de especialistas). La actividad consiste en identificar en la información facilitada diferentes argumentos a favor y en contra, y a partir de ella, tomar una decisión ante el dilema planteado. En todos los casos, los estudiantes deben justificar sus respuestas.

Fase 2: Desarrollo de un programa de instrucción en asignaturas de Didáctica de las Ciencias Experimentales en los Grados en Educación Infantil y Primaria.

Esta segunda fase comprende cuatro sesiones de 90 minutos que se desarrollan a lo largo de un cuatrimestre. En la primera sesión, se muestran ejemplos donde los estudiantes ponen en juego su capacidad de argumentación como habilidad importante en el desarrollo del pensamiento crítico. A continuación, el docente resalta la importancia de argumentar en la vida diaria, en la educación en general y en la científica en particular, y explica el modelo de argumentación de Toulmin (2003) y la adaptación de Jiménez-Aleixandre (2010), así como la contribución de la argumentación al pensamiento crítico (Blanco, España y Franco-Mariscal, 2017). En esta sesión se hace hincapié en la estructura más adecuada que debe tener un buen argumento, el cual debe constar, al menos, de tres elementos esenciales, prueba, justificación y conclusión (Toulmin, 2003; Jiménez-Aleixandre, 2010). Se expone, asimismo, el esquema de progreso de la argumentación propuesto por Osborne, Henderson, MacPherson, Szu, Wild y Yao (2016), que permite avanzar en los distintos niveles de construcción de argumentos y crítica. Por último, se proponen dos tareas para que los estudiantes argumenten, que luego se analizan en detalle. En la primera deben identificar los elementos de un argumento, y en la segunda, deben hacer uso del conocimiento base y la refutación, trabajando de esta forma las ideas científicas previas y la contraargumentación.

En la segunda sesión, el docente expone y analiza un dilema socio-científico relacionado con la dieta vegana, que se presenta posteriormente en mayor profundidad.

Durante la tercera sesión se presenta a los estudiantes un juego de rol cuyo escenario se desarrolla en un programa de debate televisivo que trata una problemática real y de actua-

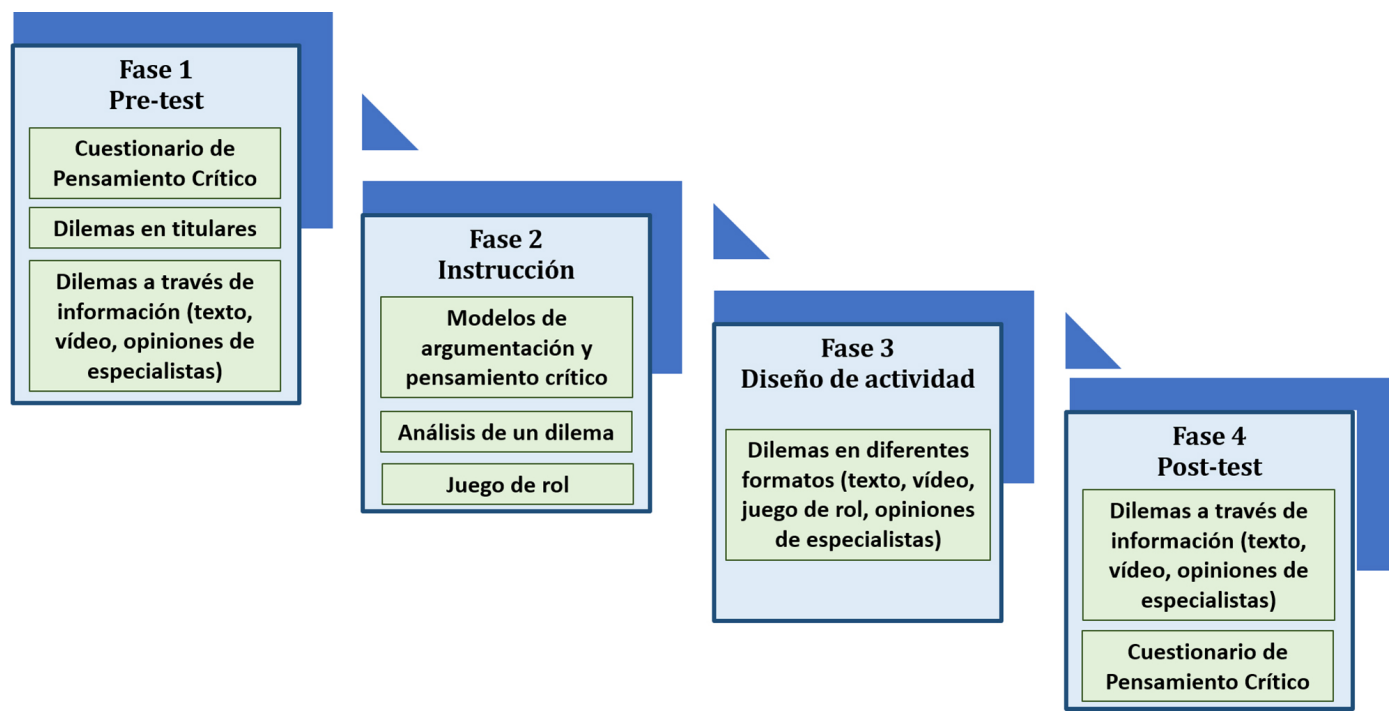

Figura 1. Fases del programa formativo 
lidad sobre el acuerdo que la Unión Europea desea establecer para eliminar el uso de plásticos en sus países integrantes en 2021 (Juárez, Hierrezuelo, Cebrián y Franco-Mariscal, 2019), disponiendo de una semana para preparar argumentos y contraargumentos para defender los diferentes roles a favor y en contra implicados en el dilema. La cuarta sesión se dedica a la escenificación del juego de rol en el aula.

\section{Fase 3: Diseño de una actividad para desarrollar habilidades de pensamiento crítico.}

En esta fase los estudiantes invierten su papel y adquieren el rol de docentes. Para ello, se les pide que diseñen una actividad adaptada a su correspondiente nivel educativo donde sus estudiantes deben trabajar la argumentación y la toma de decisiones como habilidades importantes para desarrollar el pensamiento crítico. Esta actividad forma parte del trabajo final de la asignatura y también de la evaluación. Con idea de estudiar la transferencia a la práctica está previsto realizar un seguimiento de esta temática en su fase de prácticas externas.

Fase 4: Administración de post-test para identificar el estado final de pensamiento crítico de los estudiantes a través de la argumentación.

Esta fase es idéntica a la primera, pero en este caso, los estudiantes solo responden el cuestionario de pensamiento crítico y a la segunda actividad (titulares de prensa con información) para conocer su evolución en las habilidades de pensamiento crítico.

\section{Dilema sobre la dieta vegana, ¿es saludable, sí o no?}

Esta actividad, planteada como parte de la instrucción del programa, se usó como ejemplo para analizar habilidades de argumentación y toma de decisiones en un dilema en el ámbito de la salud. La actividad plantea si la dieta vegana es o no saludable, y se presenta con el mismo esquema que las actividades administradas en el pre-test, es decir, una primera parte donde el estudiante debe elaborar argumentos a favor y en contra a partir del titular de prensa "Cada vez son más las personas que optan por la dieta vegana", y tomar una decisión, y una segunda parte de identificación de argumentos en dos textos extraídos de prensa online (ver Anexos), uno de los cuales solo presenta argumentos a favor de la dieta vegana (texto T1) y el otro en contra (texto T2), para volver a realizar una toma de decisiones. La tabla 1 recoge los argumentos a favor y en contra incluidos en ambos textos.

Tabla 1. Argumentos a favor y en contra en los textos seleccionados

\begin{tabular}{ll}
\hline Texto & Argumentos \\
\hline $\begin{array}{l}\text { Con argumentos } \\
\text { a favor (T1) }\end{array}$ & - Los alimentos veganos, especialmente los granos y frijoles ricos en fibra y \\
& las frutas y verduras envasadas con fitoquímicos, pueden ayudar a prevenir \\
& el cáncer. \\
& - Diferentes estudios muestran que comer carne de pollo, vaca y otros ani- \\
& males promueve el cáncer, mientras que comer alimentos a base de plantas \\
& puede protegernos contra ellos. \\
& - Las proteínas animales son un agente carcinógeno primordial. Los estudios \\
& en humanos también apoyan este efecto carcinogénico de la proteína animal, \\
& incluso en los niveles habituales de consumo. (Investigador epidemiológico). \\
& - Las mujeres que comen más carne tienen mayor riesgo de desarrollar cán- \\
& cer de mama (British Journal of Cancer). \\
& - Las dietas altas en grasa saturada aumentan los niveles de estrógeno, mien- \\
& tras que las dietas basadas en plantas tienden a mantener el estrógeno en un \\
& nivel seguro que no promueve el crecimiento de las células cancerosas. \\
\hline
\end{tabular}


Tabla 1. Argumentos a favor y en contra en los textos seleccionados. Continuación.

\begin{tabular}{ll}
\hline $\begin{array}{l}\text { Con argumentos } \\
\text { en contra (T2) }\end{array}$ & - La dieta vegana no solamente no es saludable, sino que no se puede seguir. \\
& que es incompatible con la vida (Catedrático de Fisiología de la Universidad \\
& de Murcia). \\
& - Las personas que siguen dieta vegana no están muertas porque toman \\
& complementos químicos, ya que sin ellos desarrollarían anemias, que si se \\
& prolongan en el tiempo producen la muerte (Catedrático de Fisiología de la \\
& Universidad de Murcia). \\
& - El $90 \%$ de los nuevos alimentos veganos son insalubres por tratarse de ali- \\
& mentos altamente procesados industrialmente y por la necesidad de suple- \\
& mentarse con vitamina B12 (Codirectora de un Centro de Nutrición). \\
& - Las dietas veganas excluyen la carne que es la mejor fuente de hierro y el \\
& hierro de las plantas se absorbe más lento que el de la carne (Doctora de Gran \\
& Bretaña).
\end{tabular}

Con idea de averiguar si el orden de los textos influía en la decisión tomada, la actividad se administró a la mitad de los estudiantes con los textos en el orden T1-T2, y a la otra mitad en el orden T2-T1. Una vez respondida la actividad, el profesor realizó un análisis pormenorizado de los argumentos dados por algunos estudiantes, y de los incluidos en cada uno de los textos, identificando las pruebas, justificación y conclusión en cada uno de ellos como los tres elementos de un buen argumento (Jiménez-Aleixandre, 2010).

Finalmente, se reflexionó sobre la importancia de tomar una decisión sin información o con ella, y los motivos del posible cambio de decisión de algunos estudiantes apoyado por alguno de los argumentos ofrecidos en los textos.

\section{Análisis de resultados}

Este trabajo realiza dos estudios.

Estudio 1. Evolución del pensamiento crítico a través de la argumentación.

Para evaluar posibles avances en el pensamiento crítico se utilizó el cuestionario de Santiuste et al. (2001) que aborda diferentes habilidades relacionadas con el pensamiento crítico, entre ellas la argumentación. Para este estudio se seleccionaron tres ítems, que se trabajaron a lo largo del programa y especialmente de forma más detallada en la tarea presentada. Concretamente, los ítems "cuando leo un texto, identifico claramente la información relevante" (ítem 3), "cuando leo un texto argumentativo, identifico claramente los argumentos que corroboran o refutan una tesis", (ítem 5) y "sé extraer conclusiones fundamentales de los textos que leo" (ítem 6), que los estudiantes debían valorar su grado de acuerdo en una escala Likert de 1 a 5 puntos. Para este estudio se compararon los valores medios obtenidos para estos ítems en el pre-test y post-test.

\section{Estudio 2. Toma de decisiones.}

Este estudio realiza tres análisis en torno a la toma de decisiones.

Un primer análisis compara los resultados obtenidos para la decisión inicial de los estudiantes, sin haber accedido a información, y su decisión final tras la lectura de los textos. Para conocer la posible existencia de diferencias estadísticamente significativas entre los dos momentos (antes y después) en cada grado, y en cada momento entre grados, 
se realizó la prueba de Chi cuadrado, considerando diferencias con una significatividad menor a 0.05 .

Un segundo análisis estudia el mantenimiento o cambio de posición de los estudiantes y la posible aparición de nuevos argumentos no relacionados con la salud que influyesen en el cambio.

Finalmente, el tercer análisis se centra en la posible influencia que tiene el orden de los textos en la decisión adoptada.

\section{Resultados}

\section{Resultados del estudio 1: Evolución del pensamiento crítico a través de la argumentación}

En general, se observó que la instrucción dada sobre argumentación favoreció a la construcción de argumentos que incluían los tres elementos esenciales del modelo de JiménezAleixandre (2010). A modo de ejemplo, se muestra el siguiente argumento dado por un estudiante, resaltando las pruebas en letra normal, la justificación en cursiva y la conclusión subrayada.

"Considero que, aunque, la dieta vegetariana y vegana es rica en fibra dietética, vitaminas $C$ y E, ácido fólico, magnesio, ácidos grasos polinsaturados... todos ellos procedentes de legumbres, cereales, frutas y verduras... en el caso de los lácteo-vegetarianos también se incluyen los huevos y lácteos. Pero, hay estudios que muestran que en su dieta existen niveles ligeramente bajos de colesterol, grasas saturadas, vitamina B12 (sustancia necesaria para el desarrollo del sistema nervioso y membranas acuosas), y menor densidad ósea (debido a la baja ingesta de calcio). Todos estos datos pueden variar en función del estado metabólico y edad de la persona y los suplementos que cubren las carencias energéticas son antinaturales, por lo que concluyo que no es saludable. (GP50)"

La tabla 2 recoge los valores medios obtenidos, antes y después del programa, en los tres ítems relacionados con la argumentación, incluidos en el cuestionario de pensamiento crítico, y que se trabajaron especialmente en esta tarea.

Tabla 2. Valores medios y desviación estándar obtenidos sobre la evolución del pensamiento crítico

\begin{tabular}{lcccc}
\hline \multicolumn{1}{c}{ Ítem } & \multicolumn{2}{c}{ Media (Pre-test) } & \multicolumn{2}{c}{ Media (Post-test) } \\
\hline & $\begin{array}{c}\text { Grado E. } \\
\text { Infantil }\end{array}$ & $\begin{array}{c}\text { Grado E. } \\
\text { Primaria }\end{array}$ & $\begin{array}{c}\text { Grado E. } \\
\text { Infantil }\end{array}$ & $\begin{array}{c}\text { Grado E. } \\
\text { Primaria }\end{array}$ \\
$\begin{array}{l}\text { 3. Cuando leo un texto, identifico } \\
\text { claramente la información relevante }\end{array}$ & $4.10 \pm 0.61$ & $4.21 \pm 0.56$ & $4.18 \pm 0.59$ & $4.32 \pm 0.68$ \\
$\begin{array}{l}\text { 5. Cuando leo un texto argumenta- } \\
\text { tivo, identifico claramente los argu- } \\
\text { mentos que corroboran o refutan } \\
\text { una tesis }\end{array}$ & $3.82 \pm 0.59$ & $4.00 \pm 0.54$ & $4.12 \pm 0.66$ & $3.97 \pm 0.51$ \\
$\begin{array}{l}\text { 6. Sé extraer conclusiones funda- } \\
\text { mentales de los textos que leo }\end{array}$ & $3.94 \pm 0.68$ & $4.02 \pm 0.56$ & $4.06 \pm 0.62$ & $4.23 \pm 0.61$ \\
\hline
\end{tabular}

Como se aprecia en la tabla 2, aunque los estudiantes de ambos grados presentan inicialmente buenas habilidades de argumentación escrita en los tres ítems estudiados (valores medios entre 3.82 y 4.21 sobre 5 puntos), son ligeramente mejores en los estudiantes del GP (valores medios superiores a 4.00). 
La comparación de estos datos con los obtenidos tras el programa, algo superiores en todos los casos, salvo en el ítem 5 para GP, parece indicar que esta actividad contribuyó a que los estudiantes de ambos grados mejorasen estas habilidades del pensamiento crítico a través de la argumentación. Aunque, bien es cierto, que esta actividad trabajó de forma pormenorizada estas habilidades en el aula, no podemos afirmar de forma taxativa que sea la causante exclusiva de esta mejora ya que formaba parte de un programa más amplio, donde se incluían otras actividades en la misma línea.

\section{Resultados del estudio 2: Toma de decisiones}

Decisión inicial y final de los estudiantes

La tabla 3 presenta los resultados obtenidos para la toma de decisión antes y después de la lectura de los textos.

Tabla 3. Toma de decisión en los dos momentos

\begin{tabular}{lcccc}
\hline & \multicolumn{2}{c}{$\begin{array}{c}\text { Grado en Educación Infantil } \\
(\mathrm{N}=111)\end{array}$} & \multicolumn{2}{c}{$\begin{array}{c}\text { Grado en Educación Primaria } \\
(\mathrm{N}=80)\end{array}$} \\
\cline { 2 - 5 } Decisión & Sí es saludable & No es saludable & Sí es saludable & No es saludable \\
\hline Antes de lectura & $36.0 \%$ & $64.0 \%$ & $47.5 \%$ & $52.5 \%$ \\
Después de lectura & $31.5 \%$ & $68.5 \%$ & $45.0 \%$ & $55.0 \%$ \\
\hline
\end{tabular}

Como se aprecia (tabla 3), los resultados en los dos momentos dentro de cada grado son similares, como confirmó la prueba de Chi cuadrado mostrando la no existencia de diferencias estadísticamente significativas $\left(\chi^{2}=0.503, p=0.478\right.$ para Gl; $\chi^{2}=0.100, p=0.751$, para GP).

Sin embargo, la decisión inicial tomada por los estudiantes del GI y GP es diferente. Así, mientras que para los estudiantes del GP no está demasiado claro si la dieta vegana es o no saludable (resultados similares en torno al 50\%), los estudiantes del Gl se inclinan principalmente hacia una dieta no saludable $(64,0 \%)$. No obstante, la prueba Chi cuadrado muestra que estas diferencias no son estadísticamente significativas $\left(\chi^{2}=2.529\right.$, $p=0.112$.

Tras disponer de la información proporcionada por la lectura, se observó en ambos grados un ligero aumento en la decisión de que la dieta vegana no es saludable, aunque en ningún caso este aumento superó el $5 \%$. La comparación de los resultados entre Gl y GP correspondientes a la decisión final tampoco reveló la existencia de diferencias estadísticamente significativas $\left(\chi^{2}=3.611, p=0.057\right)$.

La tabla 4 recoge algunas de las aportaciones realizadas por los estudiantes de ambos grados para justificar si consideraban saludable o no un estilo de vida basado en la dieta vegana.

Tabla 4. Algunos argumentos dados por los estudiantes para justificar su decisión

\begin{tabular}{l}
\hline Argumentos para justificar una dieta vegana saludable \\
\hline "La dieta vegana es saludable debido a que se sustituyen las proteínas animales por las vegeta- \\
les, por lo que al final se termina comiendo las mismas proteínas pero sin tomar grasa animal." \\
(GP34) \\
"La dieta vegana es saludable ya que aporta al organismo todos los nutrientes necesarios para su \\
funcionamiento." (GI57)
\end{tabular}


Tabla 4. Algunos argumentos dados por los estudiantes para justificar su decisión. Continuación

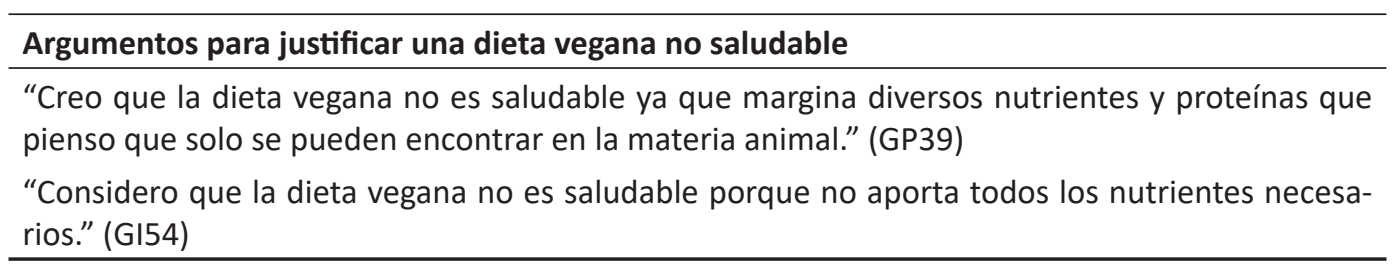

\section{Mantenimiento o cambio de postura de los estudiantes}

La figura 2 representa el porcentaje de estudiantes que se mantiene en su postura (a favor o en contra) antes y después de la lectura de los textos, o por el contrario, cambia su decisión.

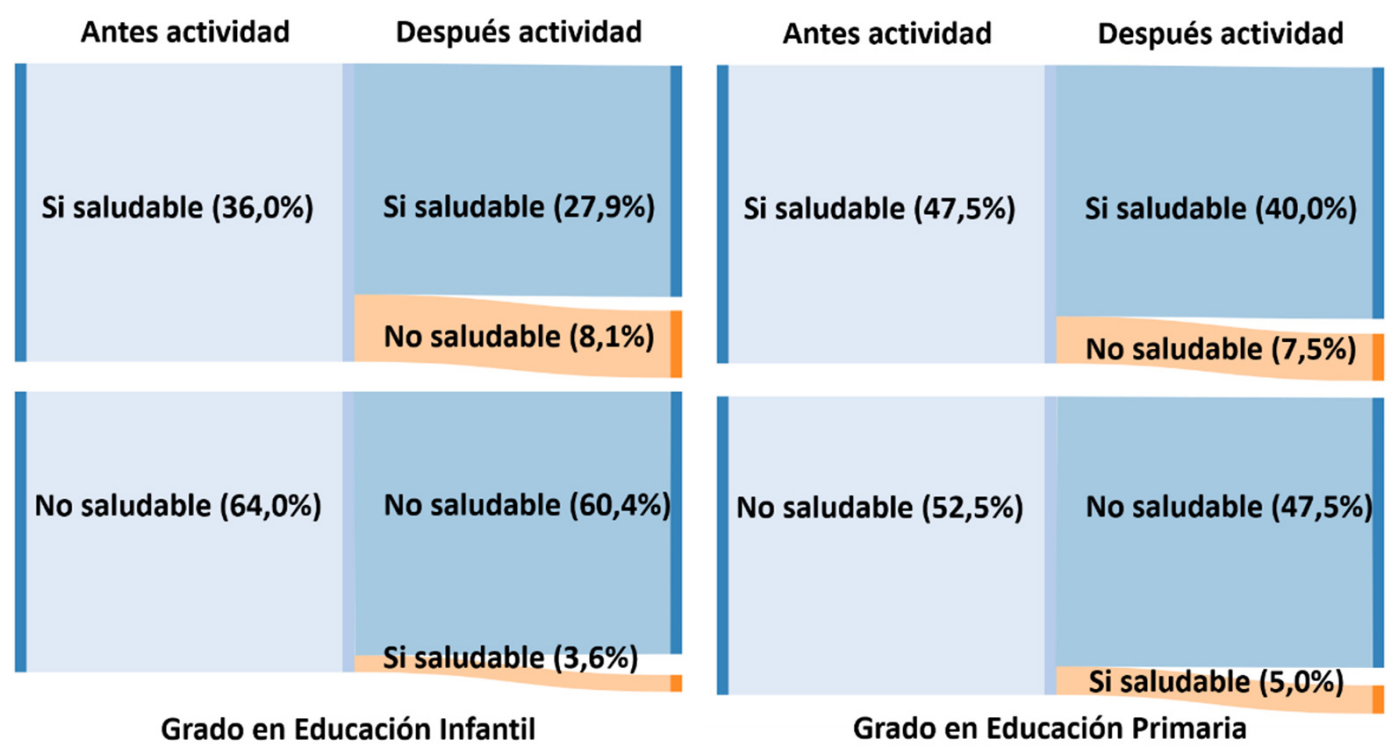

Figura 2. Mantenimiento y cambio de decisión de los estudiantes del GI y GP antes y después de la actividad

En general, se observa que los estudiantes son reacios a cambiar de postura, puesto que sólo eligen otra decisión tras la actividad el $11.7 \%$ de los estudiantes de Gl y el $12.5 \%$ de los del GP, independientemente de si su postura inicial fuese a favor o en contra del dilema. En ambos grados el cambio mayoritario se produce en los estudiantes que inicialmente indicaron que esta dieta sí era saludable y finalmente cambiaron su decisión a no saludable (figura 2).

Aunque en esta actividad, la toma de decisiones se ha establecido en base a evidencias a favor y en contra únicamente desde el punto de vista de la salud, su riqueza dimensional podría plantear la aparición de otros posibles motivos (personales, culturales, medioambientales, económicos y/o políticos) que habrían podido también influir para argumentar su cambio de postura. Tras analizar las respuestas de los estudiantes que cambiaron su postura e independientemente del sentido de ese cambio (saludable-no saludable, o viceversa), en todos los casos, se observó que los argumentos ofrecidos hacían referencia exclusivamente a motivos de salud, y en ningún caso tras el cambio se aportaron nuevos aspectos. La tabla 5 recoge varios ejemplos de estudiantes que cambiaron su postura, donde se pone de manifiesto la idea planteada. 
Tabla 5. Ejemplos de respuestas de estudiantes que cambiaron su postura

\begin{tabular}{|c|c|c|c|}
\hline \multicolumn{2}{|c|}{ Cambio de postura (Sí a No) } & \multicolumn{2}{|c|}{ Cambio de postura (No a Sí) } \\
\hline $\begin{array}{l}\text { Sí es saludable } \\
\text { (inicial) }\end{array}$ & $\begin{array}{l}\text { No es saludable } \\
\text { (final) }\end{array}$ & $\begin{array}{c}\text { No es saludable } \\
\text { (inicial) }\end{array}$ & $\begin{array}{l}\text { Sí es saludable } \\
\text { (final) }\end{array}$ \\
\hline \multicolumn{4}{|c|}{ Grado en Educación Infantil } \\
\hline $\begin{array}{l}\text { Las personas veganas } \\
\text { ingieren alimentos } \\
\text { que proceden de la } \\
\text { tierra e intentan evitar } \\
\text { los alimentos procesa- } \\
\text { dos que pueden dañar } \\
\text { la salud (GI78) }\end{array}$ & $\begin{array}{l}\text { Porque tiene caren- } \\
\text { cias, la dieta saluda- } \\
\text { ble es una dieta equi- } \\
\text { librada en la que no } \\
\text { se coma exceso de } \\
\text { carne, ni productos } \\
\text { procesados (GI78) }\end{array}$ & $\begin{array}{l}\text { Porque los veganos } \\
\text { no obtienen los } \\
\text { nutrientes necesa- } \\
\text { rios que necesita el } \\
\text { cuerpo (GI13) }\end{array}$ & $\begin{array}{l}\text { Porque ayuda a la } \\
\text { prevención contra el } \\
\text { cáncer y encontramos } \\
\text { alimentos que cubra } \\
\text { nuestra dieta y nos } \\
\text { aporten los nutrientes } \\
\text { necesarios (GI13) }\end{array}$ \\
\hline \multicolumn{4}{|c|}{ Grado en Educación Primaria } \\
\hline $\begin{array}{l}\text { Aporta todos los nu- } \\
\text { trientes necesarios } \\
\text { para nuestro cuerpo, } \\
\text { excepto una vitamina } \\
\text { (GP50) }\end{array}$ & $\begin{array}{l}\text { Los productos vega- } \\
\text { nos no completan } \\
\text { los requerimientos } \\
\text { básicos, ya que se } \\
\text { eliminan productos } \\
\text { para evitar contrain- } \\
\text { dicaciones y tienen } \\
\text { que suplementarse } \\
\text { con productos quími- } \\
\text { cos (GP50) }\end{array}$ & $\begin{array}{l}\text { Porque no contie- } \\
\text { ne proteínas que } \\
\text { son esenciales para } \\
\text { nuestro cuerpo, } \\
\text { por ejemplo, la vi- } \\
\text { tamina B12 (GP20) }\end{array}$ & $\begin{array}{l}\text { Porque baja la proba- } \\
\text { bilidad de poseer cán- } \\
\text { cer, ya que hay estu- } \\
\text { dios que recogen que } \\
\text { es beneficioso para la } \\
\text { salud y el consumo de } \\
\text { carne no (GP20) }\end{array}$ \\
\hline
\end{tabular}

\section{Influencia del orden de los textos en la decisión final}

La tabla 6 recoge el porcentaje de estudiantes de cada grado que indica cada decisión según el orden de los textos en la actividad.

Tabla 6. Toma de decisión en función del orden de los textos

\begin{tabular}{|c|c|c|c|c|c|}
\hline & & \multicolumn{2}{|c|}{ Grado E. Infantil } & \multicolumn{2}{|c|}{ Grado E. Primaria } \\
\hline & & Sí es saludable & No es saludable & Sí es saludable & No es saludable \\
\hline \multicolumn{2}{|c|}{ Antes de la lectura } & $36.0 \%(\mathrm{~N}=111)$ & $64.0 \%(N=111)$ & $47.5 \%(\mathrm{~N}=80)$ & $52.5 \%(\mathrm{~N}=80)$ \\
\hline \multirow{2}{*}{ 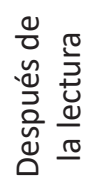 } & $\begin{array}{l}\text { Orden de } \\
\text { textos T1-T2 }\end{array}$ & $22.6 \%(N=54)$ & $77.4 \%(N=54)$ & $38.5 \%(N=39)$ & $61.5 \%(N=39)$ \\
\hline & $\begin{array}{l}\text { Orden de } \\
\text { textos T2-T1 }\end{array}$ & $38.6 \%(N=57)$ & $61.4 \%(N=57)$ & $51.2 \%(N=41)$ & $48.5 \%(N=41)$ \\
\hline
\end{tabular}

Los datos parecen revelar que existe una influencia directa en la decisión final que adoptan los estudiantes según el orden en que se le presentaron los textos. En concreto, parece que la decisión final de algunos estudiantes, independientemente del grado, siempre está más influenciada por el texto presentado en segundo lugar, independientemente de que esta información sea a favor (T1) o en contra (T2) de la dieta. Otro aspecto a resaltar es el orden en que se recibe y asimila la información, ya que parece tener más valor para una toma de decisiones la última información recibida, probablemente porque se encuentra 
almacenada en la denominada memoria activa de la persona, y puede ser utilizada inmediatamente en un corto periodo de tiempo.

Asimismo se observa una influencia más acusada cuando el orden de los textos es T1-T2, es decir, cuando el segundo texto incluía argumentos en contra, ya que para los estudiantes del GI provocó un cambio de decisión del $13.4 \%$ y para los del GP fue del $9.0 \%$, frente a un $2.6 \%$ y un $3.7 \%$, para ambos grados respectivamente cuando los textos se suministraron a la inversa (T2-T1).

\section{Consideraciones finales}

Este trabajo ha presentado los resultados de una actividad que permite a maestros en formación inicial desarrollar habilidades de pensamiento crítico a través de la argumentación y la toma de decisiones en un contexto de su vida diaria como es la dieta vegana.

Los resultados han permitido valorar la estrategia de instrucción centrada en el dilema en formato texto presentando dos informaciones sobre el mismo tema con ideas enfrentadas, uno a favor y otro en contra, como una herramienta eficaz para desarrollar pensamiento crítico. Se apoya en la mejora detectada en varias habilidades de argumentación incluidas en un cuestionario más amplio de pensamiento crítico que se administró a estudiantes del GI y GP antes y después del programa, y donde la tarea planteada pudo influir notoriamente.

A pesar de no haberse encontrado diferencias estadísticamente significativas, los resultados parecen revelar la importancia de disponer de información del tema sobre el que se desea tomar una decisión, ya que ésta puede variar, como se ha comprobado en el estudio realizado, en función de si se dispone o no de datos fiables y contrastados. Para afianzar esta hipótesis en futuros estudios sería conveniente o bien aportar más información sobre la dieta vegana, o bien dejar un tiempo en el aula para investigar al respecto. Además, otro aspecto a tener en cuenta sería analizar el sesgo de confirmación en aquellos estudiantes que no cambiaron su decisión del momento inicial al final.

Estos resultados preliminares del programa formativo sobre pensamiento crítico con maestros en formación inicial ponen de manifiesto la importancia de incorporar este tipo de actividades a los currículos de las asignaturas de Didáctica de las Ciencias Experimentales de los GI y GP. Estas permiten mejorar las capacidades argumentativas y de toma de decisiones de los futuros maestros ante problemas científicos como la energía, el medioambiente, los recursos, la salud, etc., y en definitiva mejorar su pensamiento crítico. Asimismo, pone de relieve la importancia de abordar en el aula el problema desde una dimensión integral, puesto que en este caso, los estudiantes solo tuvieron en cuenta aspectos de salud en su toma de decisiones.

Como línea de investigación futura se pretende estudiar la capacidad de los maestros en formación de transferir a su práctica educativa el conocimiento adquirido mientras realizan prácticas curriculares en los centros.

\section{Agradecimientos}

Este trabajo forma parte de los siguientes proyectos:

- Proyecto I+D+i del Plan Nacional, referencia PID2019-105765GA-I00, titulado "Ciudadanos con pensamiento crítico: Un desafío para el profesorado en la enseñanza de las ciencias", financiado por el Ministerio de Ciencia e Innovación en la convocatoria 2019. 
- Proyecto I+D de Excelencia EDU2017-82197-P, financiado por el Ministerio de Economía, Industria y Competitividad en 2017.

- Proyecto de Innovación Educativa PIE19-139 “Educación STEAM en la formación de estudiantes universitarios" financiado por la Universidad de Málaga.

\section{Referencias}

Blanco-López, A., España-Ramos, E. y Franco-Mariscal, A.J. (2016). Estrategias para mejorar la reflexión sobre la práctica de los estudiantes del Máster en Profesorado de Educación Secundaria. En P. Membiela, N. Casado y M.I. Cebreiros (Eds.), Presente y futuro de la docencia universitaria. Ourense: Educación Editora.

Blanco-López, A., España-Ramos, E. y Franco-Mariscal, A.J. (2017). Estrategias didácticas para el desarrollo del pensamiento crítico en el aula de ciencias. Ápice, Revista de Educación Científica, 1(1), 107-115. DOI: https://doi.org/10.17979/ arec.2017.1.1.2004

Cobo, C., Abril, A.M. y Romero, M. (2018). Investigando sobre el origen de los seres vivos para facilitar la comprensión de la naturaleza de la ciencia y el desarrollo del pensamiento crítico. En C. Martínez y S. García-Barros (Eds.), 28 Encuentros de Didáctica de las Ciencias Experimentales. Iluminando el cambio educativo. A Coruña: Universidade da Coruña.

Díaz, N. y Jiménez-Liso, M.R. (2012). Las controversias sociocientíficas: temáticas importancia para la educación científica. Revista Eureka sobre Enseñanza y Divulgación de las Ciencias 9(1), 54-70. DOI: http://dx.doi.org/10.25267/Rev_Eureka_ensen_ divulg_cienc.2012.v9.i1.04

Ennis, R.H. (1991). Critical thinking: a streamlined conception. Teaching Philosophy, 14(1), 5-24.

Evagorou, M., Jiménez-Aleixandre, M.P. y Osborne, J. (2012). Should we kill the grey squirrels? A study exploring students' justifications and decision-making. International Journal of Science Education, 34(3), 401-428. DOI: https://doi.org/10.1080/09500 693.2011.619211

Fonseca, Y. y Castiblanco, O. (2020). Desarrollo del pensamiento crítico y reflexivo a partir de la enseñanza del sonido. Tecné, Episteme y Didaxis, 47, 111-126. DOI: https:// doi.org/10.17227/ted.num47-7841

Jiménez-Aleixandre, M. P. (2010). 10 Ideas Clave. Competencias en argumentación y uso de pruebas, (Vol. 12). Barcelona: Graó.

Juárez-González, P., Hierrezuelo-Osorio, J. M., Cebrián-Robles, D. y Franco-Mariscal, A. J. (2019). El juego de rol como estrategia para enseñar a argumentar en ciencias. La visión de maestros en formación inicial. Aula de Innovación Educativa, 287, 15-20.

Manassero, M.A. y Vázquez, A. (2020). Evaluación de destrezas del pensamiento crítico: validación de instrumentos libres de cultura. Tecné, Episteme y Didaxis, 47, 15-32. DOI: https://doi.org/10.17227/ted.num47-9801

Osborne, J., Henderson, J. B., MacPherson, A., Szu, E., Wild, A. y Yao, S. (2016). The development and validation of a learning progression for argumentation in science. Journal of Research in Science Teaching, 53(6), 821-846. DOI: https://doi. org/10.1002/tea.21316 
Santiuste B. (Coord.), Ayala, C., Barriguete, C., García, E., Gonzales, J., Rossignoli, J., y Toledo, E. (2001). El pensamiento crítico en la práctica educativa. Madrid, España.: Fugaz Ediciones.

Simonneaux, L. y Lipp, A. (2017). Emotions, values and knowledge in students' argumentation about farm animal welfare. In ESERA 2017 Conference. Recuperado de https:// keynote.conference-services.net/resources/444/5233/pdf/ESERA2017_1071_ paper.pdf

Solbes, J. y Torres, N. (2012). Análisis de las competencias de pensamiento crítico desde el aborde de las cuestiones sociocientíficas: un estudio en el ámbito universitario. Didáctica de las Ciencias Experimentales y Sociales, 26, 247-269. DOI: https://doi. org/10.7203/DCES.26.1928

Solbes, J. y Torres, N. (2013). ¿Cuáles son las concepciones de los docentes en formación y en ejercicio sobre el pensamiento crítico? Tecné, Episteme y Didaxis, 33, 61-65.

Toulmin, S.E. (2003). The uses of argument ( $3^{\text {rd }}$ Edition). Cambridge: Cambridge University Press. 


\section{Anexo I}

Texto con argumentos a favor (T1) publicado en la revista digital PETA (https://www.peta.org/ issues/animals-used-for-food/cancer/)

\section{Cáncer}

Según la Organización Mundial de la Salud (OMS), más del 40 por ciento de todos los casos de cáncer se pueden prevenir, y la Sociedad Americana del Cáncer informa que un tercio de todas las muertes por cáncer en los Estados Unidos se puede atribuir a los hábitos alimenticios poco saludables y la falta de actividad física. Los alimentos veganos, especialmente los granos y frijoles ricos en fibra y las frutas y verduras envasadas con fitoquímicos, pueden ayudar a prevenir el cáncer. Las investigaciones sugieren que las personas que consumen alimentos veganos tienen entre 25 y 50 por ciento menos probabilidades de contraer la enfermedad. Un informe de la OMS de 2015 descubrió que el tocino, los perros calientes y otras carnes procesadas causan cáncer y que la carne roja, incluida la carne de res, cerdo y cordero, probablemente también sea cancerígena. Una serie de estudios muestra que, al comer carne de pollos, vacas y otros animales promueve el cáncer, comer alimentos a base de plantas puede protegerlo contra ellos. Aquí hay algunos de ellos: Un estudio alemán de 11 años que involucró a más de 800 hombres vegetarianos encontró que sus tasas de cáncer eran menos de la mitad de las del público en general. Un estudio de 2007 de más de 35,000 mujeres publicado en el British Journal of Cancer encontró que las mujeres que comían más carne tenían el mayor riesgo de desarrollar cáncer de mama. T. Colin Campbell, coautor del histórico estudio de China y posiblemente el investigador epidemiológico más importante del mundo, cree que las proteínas animales son un agente carcinógeno primordial. Señala que "los estudios en humanos también apoyan este efecto carcinogénico de la proteína animal, incluso en los niveles habituales de consumo". La grasa saturada también es un culpable: las dietas altas en grasa aumentan los niveles de estrógeno, mientras que las dietas basadas en plantas tienden a mantener el estrógeno en un nivel seguro que no promueve el crecimiento de las células cancerosas. Además, la fibra, que se encuentra en la mayoría de los alimentos veganos, puede ayudar a nuestros cuerpos a eliminar el exceso de estrógeno, reduciendo el riesgo de cáncer. Es fácil eliminar la carne, los huevos y los "productos lácteos" de nuestras dietas y reemplazarlos con proteínas vegetales que protegen nuestra salud en lugar de dañarla. 


\section{Anexo II}

Texto con argumentos en contra (T2) publicado en el periódico digital El Mundo (https://www. elmundo.es/papel/gastro/2018/12/20/5c1a5c9821efa0ed368b45c6.html)

\section{Veganos contra antiveganos: así será la guerra "gastro" en 2019}

Tras el boom de la comida vegana, llega el contraataque: cada vez más expertos afirman que esta dieta es dañina para la salud. Pero, como ya saben, el movimiento vegetariano no ha dejado de crecer y de extenderse. Las fuentes bien informadas nos indican que en 2010 abrió el primer restaurante vegano en la capital española, y al año siguiente algo más radical todavía, el primer crudivegano. Sin embargo, la reacción opuesta también se manifiesta hoy con más fuerza, a partir de países como Estados Unidos donde el vegetarianismo y el veganismo se extendieron mucho antes. Aquí la cuestión no es si las alcachofas son más o menos sabrosas -y lo son, y mucho-, sino si tan sólo con una dieta vegetal se preserva la salud. Y sin salud el gastronomadismo que en esta página se defiende pierde todo su sentido... En La Verdad de Murcia, Salvador Moreno, catedrático emérito de Fisiología de la universidad murciana, denuncia la impostura de la dieta vegana: «No solamente no es saludable, sino que no se puede seguir. No hay ninguna persona en el mundo que siga una dieta vegana estricta porque es incompatible con la vida ¿Entonces, por qué no están muertos? Porque toman complementos químicos, ya que sin ellos desarrollarían enfermedades importantes, tan importantes que si se prolongan en el tiempo producen la muerte. Hablamos de la anemia megaloblástica, la anemia perniciosa, etcétera». Por otro lado, Lucía Martínez, codirectora del Centro de Nutrición Aleris de Madrid, reconocía que «alrededor del $90 \%$ de los nuevos productos veganos son insalubres» por tratarse de alimentos altamente procesados industrialmente y por la necesidad de suplementarse con vitamina B12. La inquietud no es, en efecto, una exclusiva de España. Lean a la Doctora Miriam Stoppard, de Gran Bretaña, comentar la actual extensión de las deficiencias en hierro: "Las dietas que excluyen grupos enteros de alimentos, las modas alimentarias poco sanas, las dietas veganas que excluyen la mejor fuente de hierro, que es la carne, todo ello contribuye. Sí, en parte se puede culpar al incremento de dietas vegetarianas y veganas. El tipo de hierro que sólo se encuentra en la carne se absorbe mucho más fácilmente que el que hay en algunas plantas». La mesura, la moderación, parecen actitudes sensatas que merecen más seguimiento. Pero reconozcamos que, en alimentación como en lo demás, los tiempos son más de extremismo y confrontación. Así que, literalmente, iapriétense los cinturones en 2019! 
